\title{
Stretching and shearing contamination analysis for Liutex and other vortex identification methods
}

\author{
Pushpa Shrestha, Charles Nottage, Yifei Yu, Oscar Alvarez and Chaoqun Liu* (i)
}

\author{
* Correspondence: cliu@uta.edu \\ Department of Mathematics, The \\ University of Texas at Arlington, \\ Arlington, TX 76019, USA
}

\begin{abstract}
The newly developed vortex-identification method, Liutex, has provided a new systematic description of the local fluid rotation, which includes scalar, vector, and tensor forms. However, the advantages of Liutex over the other widely used vortexidentification methods such as $Q, \Delta, \lambda_{2}$, and $\lambda_{c i}$ have not been realized. These traditional methods count on shearing and stretching as a part of vortex strength. But, in the real flow, shearing and stretching do not contribute to fluid rotation. In this paper, the decomposition of the velocity gradient tensor is conducted in the Principal Coordinate for uniqueness. Then the contamination effects of stretching and shearing of the traditional methods are investigated and compared with the Liutex method in terms of mathematical analysis and numerical calculations. The results show that the Liutex method is the only method that is not affected by either stretching or shear, as it represents only the local fluid rigid rotation. These results provide supporting evidence that Liutex is the superior method over others.
\end{abstract}

Keywords: Liutex, Vorticity, Vortex, Omega method, Transition flow, Velocity gradient tensor, Principal coordinate, Principal tensor, Liutex tensor decomposition

\section{Introduction}

A vortex is recognized as the rotational motion of fluids. Within the last several decades, a lot of vortex identification methods have been developed to track the vortical structure in a fluid flow; however, we still lack unambiguous and universally accepted vortex identification criteria. This is the impediment that has caused a lot of confusions and misunderstandings in turbulence research [1]. In most research papers and textbooks, the vorticity tube/filament is regarded as a vortex and the magnitude of the vorticity as the local rotational strength. Many researchers widely acknowledged the concept of vortex defined as the vorticity concentration and other vorticity-based methods $[2,3]$ as the vorticity vector was believed to offer a mathematical definition of fluid rotational motion. Zhou and Antonia (1993) [4] utilized the spatially phasedcorrelated vorticity to characterize large-scale and organized structures in the cylinder wake. However, problems arose while applying in viscous flows, particularly in turbulent flows. In turbulent viscous flows, the rigid rotation strength is minimal near the

(c) The Author(s). 2021, corrected publication 2021 Open Access This article is licensed under a Creative Commons Attribution 4.0 International License, which permits use, sharing, adaptation, distribution and reproduction in any medium or format, as long as you give appropriate credit to the original author(s) and the source, provide a link to the Creative Commons licence, and indicate if changes were made. The images or other third party material in this article are included in the article's Creative Commons licence, unless indicated otherwise in a credit line to the material. If material is not included in the article's Creative Commons licence and your intended use is not permitted by statutory regulation or exceeds the permitted use, you will need to obtain permission directly from the copyright holder. To view a copy of this licence, visit http://creativecommons.org/licenses/by/4.0/. 
wall, where shear stress is dominant. Many researchers in the literature have supported this claim, indicating the inadequacies of vorticity-based methods. Epps (2017) [5] observed that vorticity could not distinguish a vortical region with rotational motions from a strong shear layer. Robinson (1991) [6] also uncovered the fact that the regions of strong vorticity and actual vortices are weakly related. These vorticity-based methods are the first generation (1G) of vortex identification methods as per [7], while scalar based schemes such as $\Delta, Q, \lambda_{2}, \lambda_{\mathrm{ci}}$, and $\Omega$ are known as second-generation (2G) criteria.

Attempting to remedy the problems of vorticity based methods, $2 \mathrm{G}$ vortex identification criteria such as $\Delta[8,9], Q[10], \lambda_{2}[11], \lambda_{\mathrm{ci}}[12], \Omega[13,14]$, etc., have been introduced and extensively applied in visualizing vortex structures over the past three decades. Although the 2G- schemes have been able to judge the presence of the local rotational motion to some extent, these methods need an adjustment of threshold to visualize the iso-surface plot accurately. This poses a problem as it is difficult to adjust the threshold that will define the boundary of vortical structures in a particular case [13, 15]. Moreover, the $Q$ and $\lambda_{2}$ methods are restrictive for incompressible flows due to their incompressibility assumption. These and other issues of $1 \mathrm{G}$ and $2 \mathrm{G}$ vortex identification methods prompted the development of a new vortex identification method, the Liutex method $[16,17]$, also known as a third-generation vortex identification scheme.

According to Chong and Perry (1990) [8], if the velocity gradient tensor of a threedimensional flow field has a pair of complex conjugate eigenvalues and a real eigenvalue, then the instantaneous streamline pattern presents a local swirling motion. Liu et al. (2019) [7] acknowledged that these streamlined patterns are the direct result of the real eigenvector of the velocity gradient tensor. In fact, this real eigenvector is the axis of fluid rotation which is also known as the direction of Liutex. Unlike $2 \mathrm{G}$ schemes, the Liutex method is a novel eigenvector-based method that is local, accurate, unique, and systematic. Furthermore, the systematical definition of Liutex is given as a vector which has its corresponding scalar and tensor forms [17]. The vector form of Liutex gives the direction of the local fluid rotation, while the magnitude of Liutex represents the rotational strength of a fluid rotation. Also, the Liutex vector is Galilean invariant [18-20]. According to Liu et al. [7], the Liutex method, Liutex-Omega method [16], Liutex Core Line method, and other Liutex-based methods [21-23] are the third generation (3G) of vortex identification methods. The Liutex method is discussed briefly in section 2.6.

After the introduction of the Liutex method in 2018, many doors of investigations of fluid rotations and related topics have opened. Based on Liutex definition, Gao and Liu proposed a velocity gradient tensor decomposition [24] in the rotated coordinate system, which was somewhat lengthy and cumbersome. So, later in the same year, they gave explicit expressions for the Rortex/Liutex tensor and velocity gradient tensor decomposition [25] without coordinate rotation. Recently, Yu and Liu proposed a new unique definition of Principal Coordinates and conducted a unique Principal Tensor decomposition under the Principal coordinates [26]. In this paper, the concept of Principal Coordinates and Principal Tensor decomposition is revisited briefly in section 3. Also, the issue of shear and stretching contamination of $1 \mathrm{G}$ and several $2 \mathrm{G}$ vortex identification methods is investigated in terms of mathematical analysis and numerical 
calculations. Our investigation involves calculating the velocity gradient tensor at a point inside the numerical domain (flow field). Then, a unique Principal Tensor decomposition is performed in the Principal Coordinates. In other words, the velocity gradient tensor is uniquely decomposed into its rigid rotation part, stretching part, and shearing part in the Principal Coordinates. From our investigation, it is found that $1 \mathrm{G}$ and several $2 \mathrm{G}$ vortex identification methods misinterpret shearing and stretching as a constituent of vortical structure, which is not true. Also, these methods have dimensional problems at different physical levels, which are discussed in section 4 in detail.

The organization of this paper is as follows: In Section 2, some traditional vortex identification methods are revisited including vorticity tube/filaments, $\Delta, Q, \lambda_{2}$ and $\lambda_{c i}$ methods. Section 3 describes the Liutex-based Principal Tensor Decomposition in the unique Principal Coordinates. Based on the unique Principal Tensor Decomposition in the Principal Coordinates, the theoretical contamination analysis of the vorticity, $\Delta, Q$, $\lambda_{2}, \lambda_{c i}$ and Liutex schemes are examined and presented in Section 4. Numerical experiments and their results can be found in Sections 5 and 6, respectively, and some concluding remarks are made in the last section.

\section{Review of $1 \mathrm{G}$ and $2 \mathrm{G}$ vortex identification methods}

\subsection{Vorticity-based method}

Since Helmholtz introduced the concept of vorticity tube/filament in 1858 [2], many researchers have believed that vortices consist of small vorticity tubes which are called vortex filaments, and the magnitude of vorticity gives the vortex strength. The vorticity vector is mathematically defined as the curl of the velocity. i.e.

$$
\text { Vorticity }=\nabla \times \boldsymbol{v}=\left|\begin{array}{ccc}
\boldsymbol{i} & \boldsymbol{j} & \boldsymbol{k} \\
\frac{\partial}{\partial x} & \frac{\partial}{\partial y} & \frac{\partial}{\partial z} \\
u & \nu & w
\end{array}\right|=\boldsymbol{i}\left(\frac{\partial w}{\partial y}-\frac{\partial v}{\partial z}\right)-\boldsymbol{j}\left(\frac{\partial w}{\partial x}-\frac{\partial u}{\partial z}\right)+\boldsymbol{k}\left(\frac{\partial v}{\partial x}-\frac{\partial u}{\partial y}\right)
$$

Helmholtz also defined vortex lines and vortex filaments based on the vorticity vector. According to Helmholtz, vortex lines are drawn through the fluid mass so that their direction at every point coincides with the direction of the momentary axis of rotation of the water particles lying on it. He also defined vortex filament as the portions of the fluid mass cut out from it by way of constructing corresponding vortex lines through all points of circumference of an infinitely small surface element. Another vorticity-based method to define a vortical structure is vortex-tube. Lamb [27] defines vortex-tube as: "If through every point of a small, closed curve we draw the corresponding vortex-line, we mark out a tube known as vortex-tube."

\section{$2.2 \Delta$ method}

The $\Delta$ method defines a vortex to be the region where the velocity gradient tensor $\nabla \vec{v}$ has a pair of complex conjugate eigenvalues and a real eigenvalue [8]. If $\lambda_{1}, \lambda_{2}$ and $\lambda_{3}$ are the eigenvalues of the $3 \times 3$ matrix of $\nabla \vec{v}$, then the characteristic equation can be written as

$$
\lambda^{3}+I_{1} \lambda^{2}+I_{2} \lambda+I_{3}=0
$$


where, $I_{1}, I_{2}$, and $I_{3}$ are the first, second, and third principal invariants of the characteristic eq. (2) and given by

$$
\begin{aligned}
& I_{1}=\left(\lambda_{1}+\lambda_{2}+\lambda_{3}\right)=\operatorname{tr}(\nabla \overrightarrow{\boldsymbol{v}}) \\
& I_{2}=\lambda_{1} \lambda_{2}+\lambda_{2} \lambda_{3}+\lambda_{3} \lambda_{1}=-\frac{1}{2}\left[\operatorname{tr}\left(\nabla \overrightarrow{\boldsymbol{v}}^{2}\right)-\operatorname{tr}(\nabla \overrightarrow{\boldsymbol{v}})^{2}\right] \\
& I_{3}=\lambda_{1} \lambda_{2} \lambda_{3}=\operatorname{det}(\nabla \overrightarrow{\boldsymbol{v}})
\end{aligned}
$$

The discriminant of the characteristic eq. (2) is given by

$$
\Delta=\left(\frac{\tilde{Q}}{3}\right)^{3}+\left(\frac{\tilde{R}}{2}\right)^{2}
$$

where $\tilde{Q}=I_{2}-\frac{1}{3} I_{1}{ }^{2}$ and $\tilde{R}=-I_{3}-\frac{2}{27} I_{1}{ }^{3}+\frac{1}{3} I_{1} I_{2}$.

For incompressible flow, the first principal invariant $I_{1}=0$, which results in eq. (6) becoming $\Delta=\left(\frac{I_{2}}{3}\right)^{3}+\left(\frac{I_{3}}{2}\right)^{2}$. If $\Delta \leq 0$, all three eigenvalues of $\nabla \overrightarrow{\boldsymbol{v}}$ are real, but if $\Delta>0$, there exists one real and two conjugate complex eigenvalues. The latter means that the point is inside a vortex region. Although the $\Delta$-method can capture the vortex region successfully, it is susceptible to the threshold value, which is man-made and arbitrary in general.

\section{$2.3 Q$ method}

Proposed by Hunt et al. (1988) [10], the $Q$ method is one of the most popular methods used to visualize the vortex structure. According to Chakraborty et al. (2005) [28], Q is also the second principal invariant of the velocity gradient, and the value of $Q$ can be calculated as half of the difference of squares of the Frobenius norm of the vorticity tensor and strain-rate tensor. i.e.,

$$
Q=\frac{1}{2}\left(\|\boldsymbol{B}\|_{F}^{2}-\|\boldsymbol{A}\|_{F}^{2}\right)
$$

where $\mathbf{A}$ and $\mathbf{B}$ are the symmetric and anti-symmetric part of the velocity gradient tensor, respectively.

$$
\begin{gathered}
\boldsymbol{A}=\frac{1}{2}\left(\nabla \overrightarrow{\boldsymbol{v}}+\nabla \overrightarrow{\boldsymbol{v}}^{\boldsymbol{T}}\right)=\left[\begin{array}{ccc}
\frac{\partial u}{\partial x} & \frac{1}{2}\left(\frac{\partial u}{\partial y}+\frac{\partial v}{\partial x}\right) & \frac{1}{2}\left(\frac{\partial u}{\partial z}+\frac{\partial w}{\partial x}\right) \\
\frac{1}{2}\left(\frac{\partial v}{\partial x}+\frac{\partial u}{\partial y}\right) & \frac{\partial v}{\partial y} & \frac{1}{2}\left(\frac{\partial v}{\partial z}+\frac{\partial w}{\partial y}\right) \\
\frac{1}{2}\left(\frac{\partial w}{\partial x}+\frac{\partial u}{\partial z}\right) & \frac{1}{2}\left(\frac{\partial w}{\partial y}+\frac{\partial v}{\partial z}\right) & \frac{\partial w}{\partial z}
\end{array}\right] \\
\boldsymbol{B}=\frac{1}{2}\left(\nabla \overrightarrow{\boldsymbol{v}}-\nabla \overrightarrow{\boldsymbol{v}}^{T}\right)=\left[\begin{array}{ccc}
0 & \frac{1}{2}\left(\frac{\partial u}{\partial y}-\frac{\partial v}{\partial x}\right) & \frac{1}{2}\left(\frac{\partial u}{\partial z}-\frac{\partial w}{\partial x}\right) \\
\frac{1}{2}\left(\frac{\partial v}{\partial x}-\frac{\partial u}{\partial y}\right) & 0 & \frac{1}{2}\left(\frac{\partial v}{\partial z}-\frac{\partial w}{\partial y}\right) \\
\frac{1}{2}\left(\frac{\partial w}{\partial x}-\frac{\partial u}{\partial z}\right) & \frac{1}{2}\left(\frac{\partial w}{\partial y}-\frac{\partial v}{\partial z}\right) & 0
\end{array}\right]
\end{gathered}
$$

The $Q$ method considers that a vortex occurs in the region where $Q>0$. Using the $Q$ method, it is easy to track the vortical structure by iso-surface plotting. However, $Q$ is scalar-valued, and a proper threshold, which in general does not exist, is required to 
visualize the vortex region. Also, there exists an inconsistency between the $\Delta$ method and $Q$ method as $\left(\frac{\tilde{R}}{2}\right)^{2}$ is always positive, and even if $Q<0$, it is still possible that $\Delta$ could be positive.

\section{$2.4 \lambda_{c i}$ criterion}

The $\lambda_{c i}$ criterion $[12,28]$ uses the imaginary part of the complex eigenvalues of the velocity gradient tensor to visualize the vortex structure. It is based on the idea that the local time-frozen streamlines exhibit a rotational flow pattern when $\nabla \vec{v}$ has a pair of complex conjugate eigenvalues. In this case, the tensor transformation of $\nabla \vec{v}$ is given by

$$
\nabla \overrightarrow{\boldsymbol{v}}=\left[\begin{array}{lll}
\vec{v}_{r} & \vec{v}_{c r} & \vec{v}_{c i}
\end{array}\right]\left[\begin{array}{ccc}
\lambda_{r} & 0 & 0 \\
0 & \lambda_{c r} & \lambda_{c i} \\
0 & -\lambda_{c i} & \lambda_{c r}
\end{array}\right]\left[\begin{array}{lll}
\vec{v}_{r} & \vec{v}_{c r} & \vec{v}_{c i}
\end{array}\right]^{-1}
$$

where $\lambda_{r}$ is the real eigenvalue with the corresponding real eigenvector $\vec{v}_{r}$ and the pair of complex conjugate eigenvalues are $\lambda_{c r} \pm i \lambda_{c i}$ with corresponding eigenvectors $\vec{v}_{c r} \pm i$ $\vec{v}_{c i}$. In this case, in the local curvilinear system $\left(c_{1}, c_{2}, c_{3}\right)$ spanned by the eigenvector $\left(\vec{v}_{r}, \vec{v}_{c r}, \vec{v}_{c i}\right)$, the instantaneous streamlines exhibit a spiral motion. The equations of such streamlines can be written as:

$$
\begin{aligned}
& c_{1}(t)=c_{1}(0) e^{\lambda_{r} t} \\
& c_{2}(t)=\left[c_{2}(0) \cos \left(\lambda_{c i} t\right)+c_{3}(0) \sin \left(\lambda_{c i} t\right)\right] e^{\lambda_{c r} t} \\
& c_{3}(t)=\left[c_{3}(0) \cos \left(\lambda_{c i} t\right)-c_{2}(0) \sin \left(\lambda_{c i} t\right)\right] e^{\lambda_{c r} t}
\end{aligned}
$$

Despite being used by many researchers to track the vortical structure, the strength of this swirling motion may not be quantified accurately by $\lambda_{c i}$. This is largely because the tensor transformation given by (10) is a similar transformation, but not an orthogonal transformation, since $\vec{v}_{r}, \vec{v}_{c r}, \vec{v}_{c i}$ are not orthogonal. In a non-orthogonal system, vorticity is not invariant $\left(|\nabla \times \vec{v}| \neq 2 \lambda_{c i}\right)$. As well-known that vorticity is invariant in any orthogonal transformation system and therefore, $\lambda_{c i}$ cannot be used as the strength of fluid rotation. We cannot use a coordinate transformation which changes the vorticity, and the new tensor is different from the original tensor, or $\nabla \vec{V}$ is not $\nabla \vec{v}$, making it clear that transformation used in $\lambda_{c i}$ method is not Galilean invariant. On the other hand, the Liutex transformation is an orthogonal transformation that preserves the invariance of vorticity.

\section{$2.5 \lambda_{2}$ criterion}

The $\lambda_{2}$-criterion is calculated based on the observation that in a vortical region, pressure tends to be the lowest on the axis of a swirling motion of fluid particles. This happens because the centrifugal force is balanced by the pressure force (the cyclostrophic balance). This method is valid only in a steady inviscid and incompressible flow [11]. However, this assumption fails to accurately identify vortices under compressible, unsteady, and viscous conditions. By neglecting these unsteady and viscous effects, the symmetric part $\mathbf{S}$ of the gradient of the incompressible Navier-Stokes equation can be expressed as: 


$$
\boldsymbol{S}=\boldsymbol{A}^{2}+\boldsymbol{B}^{2}=-\frac{\nabla(\nabla p)}{\rho}
$$

where $p$ is the pressure and eq. (14) is a representation of the pressure Hessian matrix.

$$
(\nabla(\nabla p))_{i j}=\frac{\partial^{2} p}{\partial x_{i} \partial y_{i}} \text {. To capture the region of local pressure minimum in a plane perpen- }
$$

dicular to the vortex core line, Jeong \& Hussain defined the vortex core as a connected region with two positive eigenvalues of the pressure Hessian matrix, i.e., a connected region with two negative eigenvalues of the symmetric tensor $\mathbf{S}$. If $\lambda_{S 1}, \lambda_{S 2} \& \lambda_{S 3}$ are three real eigenvalues of the symmetric tensor $S$ and when setting them in order in such a way that $\lambda_{S 1} \geq \lambda_{S 2} \geq \lambda_{S 3}$, there must be $\lambda_{S 2}<0$ as two eigenvalues are negative, which confirms the existence of a vortex. In general, $\lambda_{S 2}$ cannot be expressed in terms of eigenvalues of the velocity gradient tensor; however, in some special cases when eigenvectors are orthonormal, $\lambda_{S 2}$ can be exclusively determined by eigenvalues of the velocity gradient tensor. Vortex structure can be visualized as iso-surface by selecting a proper threshold of $\lambda_{S 2}$ [7]. The relation between the eigenvalues of the symmetric tensor $\mathbf{A}^{2}+\mathbf{B}^{2}$ and second invariant $Q$ is given by:

$$
Q=-\frac{1}{2} \operatorname{tr}\left(\boldsymbol{A}^{2}+\boldsymbol{B}^{2}\right)=-\frac{1}{2}\left(\lambda_{S 1}+\lambda_{S 2}+\lambda_{S 3}\right)
$$

While the Q-criterion measures the excess of vorticity rate over the strain rate magnitude in all directions, the $\lambda_{2}$ criterion looks for this excess only on a specific plane [11]. For comparative studies of different schemes, another article, "on the relationship between vortex identifications methods" by Chakraborty \& Balachandar [28] is also recommended. There is yet another article by Liu et al., "Third generation of vortex identification methods", which reviews the second generation of vortex identification methods. These 2G methods are based on the eigenvalues of $\nabla \vec{v}$. Afterward, we are presented with the novel third generation of vortex identification methods [7].

\subsection{Liutex}

Definition 1: Liutex is a vector defined as $\vec{R}=R \vec{r}$, where $R$ is the magnitude of Liutex, and $\vec{r}$ is the direction of Liutex.

According to Ref. [29], $\vec{r}$ is the normalized real eigenvector of the velocity gradient tensor such that $\vec{\omega} \cdot \vec{r}>0$ and the explicit formula of $\mathrm{R}$ is

$$
R=\vec{\omega} \cdot \vec{r}-\sqrt{(\vec{\omega} \cdot \vec{r})^{2}-4 \lambda_{c i}^{2}}
$$

Dr. Liu classified the Liutex method as the third generation of vortex identification method, while the Q method, $\lambda_{c i}$ criterion and $\lambda_{2}$ criterion are all classified as secondgeneration methods since they are all scalar and eigenvalue related. Liutex is a vector which overcomes the drawbacks of the scalar methods, e.g., it can give the direction of rotation and represents the rotational strength accurately. Liutex is finally found as a mathematical definition of fluid rigid rotation or vortex.

\section{Principal coordinates and principal decomposition}

Definition 2: The Principal Coordinate at a point is a coordinate that satisfies [26]: 
1. Its $Z$-axis is parallel to the $\vec{r}$ (direction of Liutex) and $\vec{\omega} \cdot \vec{Z}>0$

2. The velocity gradient tensor under this coordinate is in the form of:

$$
\nabla \boldsymbol{V}=\left[\begin{array}{ccc}
\lambda_{c r} & \frac{\partial U}{\partial Y} & 0 \\
\frac{\partial V}{\partial X} & \lambda_{c r} & 0 \\
\frac{\partial W}{\partial X} & \frac{\partial W}{\partial Y} & \lambda_{r}
\end{array}\right]
$$

where $\lambda_{r}$ and $\lambda_{c r}$ are the real eigenvalue and real part of the conjugate complex eigenvalue pair of the velocity gradient tensor, respectively, for rotation points.

3. $\frac{\partial U}{\partial Y}<0$ and $\left|\frac{\partial U}{\partial Y}\right| \leq\left|\frac{\partial V}{\partial X}\right|$.

4. The rotational angle of the $\mathrm{X}-\mathrm{Y}$ coordinates around the $\mathrm{Z}$-axis must be smaller than $90^{\circ}$ or $-90^{\circ}<\theta \leq 90^{\circ}$

The following two theorems are valid in the Principal Coordinate.

Theorem 1: Under the Principal Coordinates, $\frac{\partial U}{\partial Y}=-\frac{R}{2}$, where $R$ is the magnitude of Liutex.

Proof: For an arbitrary velocity gradient tensor $\nabla \boldsymbol{v}$, there exists a special orthogonal rotation matrix $Q_{r}$ that aligns the Z-axis of the new frame XYZ with the Liutex direction $\vec{r}$ after rotation.

$$
\nabla \boldsymbol{V}=\boldsymbol{Q}_{r} \nabla \boldsymbol{v} \boldsymbol{Q}_{r}^{T}=\left[\begin{array}{ccc}
\frac{\partial U}{\partial X} & \frac{\partial U}{\partial Y} & 0 \\
\frac{\partial V}{\partial X} & \frac{\partial V}{\partial Y} & 0 \\
\frac{\partial W}{\partial X} & \frac{\partial W}{\partial Y} & \frac{\partial W}{\partial Z}
\end{array}\right]
$$

Where xyz is the original frame and XYZ is the new frame after $\boldsymbol{Q}_{\boldsymbol{r}}$-rotation.

Then, we apply a second rotation $\boldsymbol{P}_{\boldsymbol{r}}$ around the new Z-axis to get the Principal Coordinates.

After the $\boldsymbol{P}_{\boldsymbol{r}}$ - rotation, the corresponding velocity gradient tensor $\nabla \boldsymbol{V}_{\boldsymbol{\theta}}$ can be written as

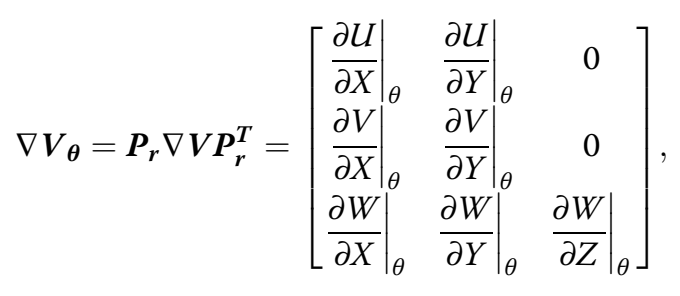

where the rotation matrix $\boldsymbol{P}_{\boldsymbol{r}}$ is given by

$$
\boldsymbol{P}_{\boldsymbol{r}}=\left[\begin{array}{ccc}
\cos \theta & \sin \theta & 0 \\
-\sin \theta & \cos \theta & 0 \\
0 & 0 & 1
\end{array}\right]
$$

and,

$$
\left.\frac{\partial U}{\partial Y}\right|_{\theta}=\alpha \sin (2 \theta+\phi)-\beta
$$




$$
\begin{aligned}
& \left.\frac{\partial V}{\partial X}\right|_{\theta}=\alpha \sin (2 \theta+\phi)+\beta \\
& \left.\frac{\partial U}{\partial X}\right|_{\theta}=-\alpha \cos (2 \theta+\phi)+\frac{1}{2}\left(\frac{\partial U}{\partial X}+\frac{\partial V}{\partial Y}\right) \\
& \left.\frac{\partial V}{\partial Y}\right|_{\theta}=\alpha \cos (2 \theta+\phi)+\frac{1}{2}\left(\frac{\partial U}{\partial X}+\frac{\partial V}{\partial Y}\right) \\
& \alpha=\frac{1}{2} \sqrt{\left(\frac{\partial v}{\partial y}-\frac{\partial u}{\partial x}\right)^{2}+\left(\frac{\partial v}{\partial x}+\frac{\partial u}{\partial y}\right)^{2}} \\
& \beta=\frac{1}{2}\left(\frac{\partial v}{\partial x}-\frac{\partial u}{\partial y}\right) \\
& \phi=\left\{\begin{array}{c}
\arctan \left(\frac{\partial x}{\frac{\partial v}{\partial y}-\frac{\partial u}{\partial x}}\right), \frac{\partial v}{\partial y}-\frac{\partial u}{\partial x} \neq 0 \\
\frac{\pi}{2}, \frac{\partial u}{\partial y}=0, \frac{\partial v}{\partial x}+\frac{\partial u}{\partial y}>0 \\
-\frac{\pi}{2}, \frac{\partial v}{\partial y}-\frac{\partial u}{\partial x}=0, \frac{\partial v}{\partial x}+\frac{\partial u}{\partial y}<0
\end{array}\right.
\end{aligned}
$$

Then, according to Liu et al., (2019) [7] the Liutex magnitude/rotational strength is defined as

$$
R=\left\{\begin{array}{c}
2(|\beta|-\alpha), \beta^{2}>\alpha^{2} \\
0, \text { otherwise }
\end{array}\right.
$$

From part (2) in Def. 2,

$$
\left.\frac{\partial U}{\partial X}\right|_{\theta}=\left.\frac{\partial V}{\partial Y}\right|_{\theta}
$$

thus,

$$
\cos (2 \theta+\phi)=0
$$

Which implies $\sin (2 \theta+\phi)=1$ or -1 . This leads us to the following four cases.

Case $1: \beta>0$ and $\sin (2 \theta+\phi)=1$

$$
\left.\frac{\partial U}{\partial Y}\right|_{\theta}=\alpha-\beta=-\frac{1}{2} R
$$

Case 2: $\beta>0$ and $\sin (2 \theta+\phi)=-1$

$$
\begin{aligned}
& \left.\frac{\partial U}{\partial Y}\right|_{\theta}=-\alpha-\beta \\
& \left.\frac{\partial V}{\partial X}\right|_{\theta}=-\alpha+\beta
\end{aligned}
$$

However,

$$
|-\alpha-\beta|>|-\alpha+\beta|
$$

This contradicts part 3 in Def. 2, therefore, $\sin (2 \theta+\phi) \neq-1$. 
Case 3: $\beta<0$ and $\sin (2 \theta+\phi)=1$

$$
\begin{aligned}
& \left.\frac{\partial U}{\partial Y}\right|_{\theta}=\alpha-\beta \\
& \left.\frac{\partial V}{\partial X}\right|_{\theta}=\alpha+\beta
\end{aligned}
$$

However,

$$
|\alpha-\beta|>|\alpha+\beta|
$$

This contradicts part 3 in Def. 2, therefore, $\sin (2 \theta+\phi) \neq 1$.

Case $4: \beta<0$ and $\sin (2 \theta+\phi)=-1$

$$
\left.\frac{\partial U}{\partial Y}\right|_{\theta}=\alpha-\beta=-\frac{1}{2} R
$$

Theorem 2: For an arbitrary velocity gradient tensor with one real eigenvalue and two conjugated complex eigenvalues, the Principal Coordinate is unique.

Proof: From the proof of theorem 1, if $\beta>0$, then $\cos (2 \theta+\phi)$ and $\sin (2 \theta+\phi)$ must be 0 and 1 , respectively. Thus, $2 \theta+\phi=\frac{\pi}{2} \Rightarrow \theta=\frac{1}{2}\left(\frac{\pi}{2}-\phi\right)$. Similarly, if $\beta<0$, then $\cos (2 \theta+$ $\phi)$ and $\sin (2 \theta+\phi)$ must be 0 and -1 , respectively. So, $2 \theta+\phi=\frac{3 \pi}{2} \Rightarrow \theta=\frac{1}{2}\left(\frac{3 \pi}{2}-\phi\right)$. Therefore, the angle of rotation around the $\mathrm{Z}$-axis is unique, and so is the Principal Coordinate.

Note that if X-and Y-axes are rotated by additional $180^{\circ}$, the new coordinates are still satisfied with the principal coordinate definition. However, since we limit the Protation angle to be less than $90^{\circ}$ or $-90^{\circ}<\theta \leq 90^{\circ}$. Under such a limit, the Principal Coordinate is unique.

Definition 3: The Principal Decomposition is the decomposition of velocity gradient tensor under the Principal Coordinates i.e.

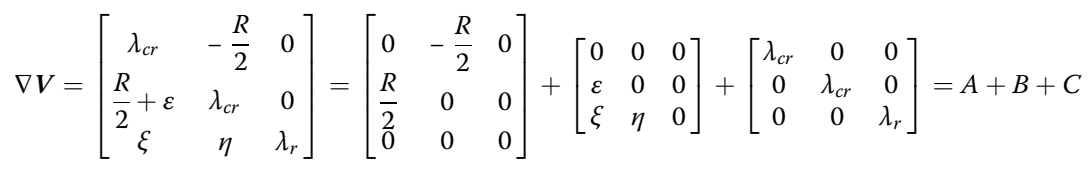

Here A represents the rotation part, B represents the shear part, and C represents the stretching part.

Mathematically, the popular Cauchy-Stokes decomposition is correct, but the physical meaning is dubious. For instance, according to the Cauchy-Stokes decomposition, the velocity gradient tensor $\left[\begin{array}{cc}0 & 2 y \\ 0 & 0\end{array}\right]$ decomposes to anti-symmetric tensor $\left[\begin{array}{cc}0 & y \\ -y & 0\end{array}\right]$ and symmetric tensor $\left[\begin{array}{ll}0 & y \\ y & 0\end{array}\right]$. This indicates that shear flow has rotation. But, in general, the shear flow does not represent fluid rotation. Also, the Cauchy-Stokes decomposition depends on the choice of coordinates, and hence it is not Galilean invariant. Yu et al. [26] used the Cauchy-Stokes decomposition to decompose the velocity gradient tensor into the rotation, deformation, and stretching matrices and found out that the norm of rotation, symmetric deformation, and stretching matrices are all different, except for 2-norm, under different coordinates. Then, a question arose. Under which coordinates, does the Cauchy-Stokes tensor 
decomposition give the right stretching (compression) and deformation? In other words, is it possible to decompose a velocity gradient tensor into the rotation, deformation, and stretching matrices without being affected by a coordinate change? Yu et.al [26] presume that there must be a unique coordinate that can give a unique tensor decomposition for stretching (compression), deformation, shear, and rotation. They call these coordinates as the "Principal Coordinates" and tensor decomposition as the "Principal Tensor Decomposition". The Principal Decomposition, being Galilean invariant, correctly and uniquely decomposes the velocity gradient tensor into its rotation part, shear part, and stretching part. This decomposition expresses that the shear and stretching contamination analysis can only be uniquely done in the Principal Coordinates as decomposition in Principal Coordinates is not affected by coordinate changes. The detailed information about the Principal Coordinates and Principal Tensor Decomposition can be found in reference [26].

\section{Theoretical contamination analysis}

Kolar et al [32] numerically investigated the stretching response of several popular vortex identification methods and found that Liutex does not respond to uniaxial stretching coupled with an inevitable uniform radial contraction for incompressible flow. In this paper, we give mathematical expression that shows the effect of strectching and shearing on several vortex identification methods. Also, these effects are presented numerically.The velocity gradient tensor, $\nabla \vec{V}=\left[\begin{array}{ccc}\lambda_{c r} & -\frac{R}{2} & 0 \\ \frac{R}{2}+\varepsilon & \lambda_{c r} & 0 \\ \xi & \eta & \lambda_{r}\end{array}\right]$ in Principal Coordinates, which shows the relation between Liutex and $\lambda_{c i}$ is $\lambda_{c i}=\sqrt{\frac{R}{2}\left(\frac{R}{2}+\varepsilon\right)}$. Apparently, $\lambda_{c i}$ is contaminated by $\varepsilon$ (shear). The vortex structure drawn by Liutex and $\lambda_{c i}$ are quite different and detailed analysis can be found in [17]. $\lambda_{c i}$ considers shear deformation $\varepsilon$ as a part of the fluid rotation and thus may not represent the pure/rigid rotational strength of fluid motion correctly. A detailed explanation with mathematical expression about how shear affects $\lambda_{c i}$ is given later in chapter 4.3. $\lambda_{c i}$ is also characterized as a scalar-valued criterion [7].

The velocity gradient tensor in the Principal Coordinate is:

$$
\nabla \overrightarrow{\boldsymbol{V}}=\left[\begin{array}{ccc}
\lambda_{c r} & -\frac{1}{2} R & 0 \\
\frac{1}{2} R+\varepsilon & \lambda_{c r} & 0 \\
\xi & \eta & \lambda_{r}
\end{array}\right]
$$

Then the Principal Decomposition is given by

$$
\nabla \overrightarrow{\boldsymbol{V}}=\left[\begin{array}{ccc}
\lambda_{c r} & -\frac{1}{2} R & 0 \\
\frac{1}{2} R+\varepsilon & \lambda_{c r} & 0 \\
\xi & \eta & \lambda_{r}
\end{array}\right]=\left[\begin{array}{ccc}
0 & -\frac{R}{2} & 0 \\
R & 0 & 0 \\
\frac{2}{2} & 0 & 0
\end{array}\right]+\left[\begin{array}{ccc}
0 & 0 & 0 \\
\varepsilon & 0 & 0 \\
\xi & \eta & 0
\end{array}\right]+\left[\begin{array}{ccc}
\lambda_{c r} & 0 & 0 \\
0 & \lambda_{c r} & 0 \\
0 & 0 & \lambda_{r}
\end{array}\right]=A+B+C
$$

A is the rotation part, B is the shear part and $\mathrm{C}$ is the stretching part.

For the first time in the literature, we are defining the several vortex identification schemes in the Principal Coordinate, which will allow us to analyze the contamination effects on these traditional vortex identification methods. 


\subsection{Contamination of vorticity}

Plugging in the gradient components from (40) into the eq. (1) gives us the vorticity vector as

$$
\boldsymbol{\omega}=(\eta,-\xi, R+\varepsilon)^{T}
$$

and its magnitude is

$$
\|\boldsymbol{\omega}\|=\sqrt{\eta^{2}+\xi^{2}+(R+\varepsilon)^{2}}
$$

From the eqs. (42) and (43), it can be concluded that a vorticity vector does not only represent rotation but also claims shearing and stretching components to be a part of the vortical structure, which is clearly contaminated by shears.

\subsection{Contamination of $\mathrm{Q}$ method}

First, we perform the Cauchy-Stokes decomposition on the Principal Coordinate. Then, the scalar magnitude of the Q method can be calculated based on the definition provided by eq. (7).

$$
\begin{aligned}
\nabla \overrightarrow{\boldsymbol{V}} & =\left[\begin{array}{ccc}
\lambda_{c r} & -\frac{1}{2} R & 0 \\
\frac{1}{2} R+\varepsilon & \lambda_{c r} & 0 \\
\xi & \eta & \lambda_{r}
\end{array}\right]=\left[\begin{array}{ccc}
\lambda_{c r} & \frac{1}{2} \varepsilon & \frac{1}{2} \xi \\
\frac{1}{2} \varepsilon & \lambda_{c r} & \frac{1}{2} \eta \\
\frac{1}{2} \xi & \frac{1}{2} \eta & \lambda_{r}
\end{array}\right]+\left[\begin{array}{ccc}
0 & -\frac{1}{2} R-\frac{1}{2} \varepsilon & -\frac{1}{2} \xi \\
\frac{1}{2} R+\frac{1}{2} \varepsilon & 0 & -\frac{1}{2} \eta \\
\frac{1}{2} \xi & \frac{1}{2} \eta & 0
\end{array}\right]=A_{Q}+\boldsymbol{B}_{Q} \\
Q & =\frac{1}{2}\left(\left\|\boldsymbol{B}_{Q}\right\|_{F}^{2}-\left\|\boldsymbol{A}_{\mathbf{Q}}\right\|_{F}^{2}\right) \\
& =\frac{1}{2}\left[2\left(\frac{R}{2}+\frac{\varepsilon}{2}\right)+2\left(\frac{\xi}{2}\right)^{2}+2\left(\frac{\eta}{2}\right)^{2}\right]-\frac{1}{2}\left[2 \lambda_{c r}{ }^{2}+\lambda_{r}{ }^{2}+2\left(\frac{\varepsilon}{2}\right)^{2}+2\left(\frac{\xi}{2}\right)^{2}+2\left(\frac{\eta}{2}\right)^{2}\right] \\
& =\left(\frac{R}{2}\right)^{2}+\frac{1}{2} R \cdot \varepsilon-\lambda_{c r}{ }^{2}-\frac{1}{2} \lambda_{r}{ }^{2}
\end{aligned}
$$

In the expression of $\mathrm{Q}$ above, there are not only $\mathrm{R}$ (the magnitude of rotation) but also $\varepsilon, \lambda_{c r}$ and $\lambda_{r}$, which are components of either the shear part or stretching part. Therefore, the value of $\mathrm{Q}$ is undoubtedly contaminated by shear and stretching. Also, $Q$ contains an $R^{2}$ term, indicating dimensional distortion of a fluid rotation.

\subsection{Contamination of $\lambda_{c i}$ criterion}

The characteristic equation of velocity gradient tensor given by (40) is

$$
\left(\lambda-\lambda_{r}\right)\left[\left(\lambda-\lambda_{c r}\right)^{2}+\frac{R}{2}\left(\frac{R}{2}+\varepsilon\right)\right]=0
$$

Thus, the eigenvalues are.

$$
\lambda_{1}=\lambda_{r}, \lambda_{2}=\lambda_{c r}+i \sqrt{R / 2(R / 2+\varepsilon)}, \lambda_{3}=\lambda_{c r}-i \sqrt{R / 2(R / 2+\varepsilon .)}
$$

Since rotation is orthogonal, eigenvalues are the same as the original velocity gradient tensor,

$$
\lambda_{2}=\lambda_{c r}+i \sqrt{R / 2(R / 2+\varepsilon)}=\lambda_{c r}+i \lambda_{c i}
$$




$$
\lambda_{3}=\lambda_{c r}-i \sqrt{R / 2(R / 2+\varepsilon)}=\lambda_{c r}-i \lambda_{c i}
$$

Therefore, we have

$$
\frac{R}{2}\left(\frac{R}{2}+\varepsilon\right)=\lambda_{c i}^{2}
$$

Thus,

$$
\lambda_{c i}=\sqrt{\frac{R}{2}\left(\frac{R}{2}+\varepsilon\right)}
$$

Despite being dimensionally correct, the expression of $\lambda_{c i}$ has $\varepsilon$, which is a component of the shear part and thus is contaminated by shear.

\subsection{Contamination of $\Delta$ method}

In section 4.2, the three roots of the characteristic equation are

$$
\lambda_{1}=\lambda_{r}, \lambda_{2}=\lambda_{c r}+i \sqrt{R / 2(R / 2+\varepsilon)}, \lambda_{3}=\lambda_{c r}-i \sqrt{R / 2(R / 2+\varepsilon)}
$$

Plug their values into (2),(3), and (4)

$$
\begin{aligned}
& I_{1}=\lambda_{1}+\lambda_{2}+\lambda_{3}=\lambda_{r}+2 \lambda_{c r} \\
& I_{2}=\lambda_{1} \lambda_{2}+\lambda_{2} \lambda_{3}+\lambda_{3} \lambda_{1}=2 \lambda_{r} \lambda_{c r}+\lambda_{c r}^{2}+\frac{R}{2}\left(\frac{R}{2}+\varepsilon\right) \\
& I_{3}=\lambda_{1} \lambda_{2} \lambda_{3}=\lambda_{r}\left[\lambda_{c r}^{2}+\frac{R}{2}\left(\frac{R}{2}+\varepsilon\right)\right] \\
& \tilde{Q}=I_{2}-\frac{1}{3} I_{1}{ }^{2}=-\frac{1}{3}\left(\lambda_{c r}-\lambda_{r}\right)^{2}+\frac{R}{2}\left(\frac{R}{2}+\varepsilon\right) \\
& \tilde{R}=-I_{3}-\frac{2}{27} I_{1}^{3}+\frac{1}{3} I_{1} I_{2}=\frac{2}{27}\left(\lambda_{c r}-\lambda_{r}\right)^{3}+\frac{2}{3}\left(\lambda_{c r}-\lambda_{r}\right) \frac{R}{2}\left(\frac{R}{2}+\varepsilon\right)
\end{aligned}
$$

Then, the expression for $\Delta$ can be written as

$$
\begin{aligned}
\Delta & =\left(\frac{\tilde{Q}}{3}\right)^{3}+\left(\frac{\tilde{R}}{2}\right)^{2} \\
& =\frac{1}{243}\left[9\left(\frac{R}{2}\right)^{3}\left(\frac{R}{2}+\varepsilon\right)^{3}-6\left(\frac{R}{2}\right)^{2}\left(\frac{R}{2}+\varepsilon\right)^{2}\left(\lambda_{c r}-\lambda_{r}\right)^{2}+\frac{5 R}{2}\left(\frac{R}{2}+\varepsilon\right)\left(\lambda_{c r}-\lambda_{r}\right)^{4}\right]
\end{aligned}
$$

The expression of $\Delta$ includes $\varepsilon, \lambda_{r}$ and $\lambda_{c r}$, indicating the contamination of $\Delta$ method by shear and stretching. Moreover, $\Delta$ contains the sixth power of $R$, which confirms that it cannot accurately denote rotation strength.

Next, we investigate the contamination of these schemes with the help of a numerical example.

\section{Vortex example: Burgers vortex}

In this test case, Burgers vortex is examined to justify the comparison of the effects of shearing and stretching/compression on different criteria. The Burgers vortex is an exact steady solution of the Navier-Stokes equation that can be used to model fine scales of 
turbulence [30]. The Burgers vortex forms when an inward radial flow concentrates and spins around the symmetric axis and the flow moves out in both directions along the $\mathrm{z}$ axis [31]. The velocity gradient tensor is obtained from the Burgers vortex.

The velocity components of Burgers vortex in the cylindrical coordinate system are given by:

$$
\begin{aligned}
& v_{r}=-\xi r \\
& v_{\theta}=\frac{\Gamma}{2 \pi r}\left(1-e^{\frac{-r^{2} \xi}{2 v}}\right) \\
& v_{z}=2 \xi z
\end{aligned}
$$

where $\Gamma$ is the circulation that characterizes the intensity of the vortex and $\xi$ is another constant representing the axisymmetric strain rate, $v$ the kinematic viscosity, and $r$ is the distance of the chosen point from the centerline in the Burgers vortex.

For post-processing, the velocity components are converted into the Cartesians coordinate system given by:

$$
\begin{aligned}
& u=-\xi x-\frac{\Gamma}{2 \pi r^{2}}\left(1-e^{\frac{-r^{2} \xi}{2 v}}\right) y \\
& v=-\xi y+\frac{\Gamma}{2 \pi r^{2}}\left(1-e^{\frac{-r^{2} \xi}{2 v}}\right) x \\
& w=2 \xi z
\end{aligned}
$$

The existence of the vortex structure is highly contingent on the selection of parameters. For the proper vortex structure visualization, we take dimensionless quantity $\xi=$ $0.042, \Gamma=1.45$ and $v=0.01$. The calculation domain is limited to $50 \times 20 \times 20$ grid points with a step size of 0.5 due to the limited computational memory. The vortex strength is strong in the core and becomes weak when moving away from the center. The streamlines of the Burgers vortex exhibit a spiral pattern around the vortex rotation axis line which is demonstrated in Fig. 1 a and b, which show that flows enter from a radial direction and stretches outward spinning around the axis.

\section{Numerical contamination analysis}

The velocity gradient tensor in the Principal Coordinate is:

$$
\nabla \boldsymbol{V}=\left[\begin{array}{lll}
\frac{\partial u}{\partial x} & \frac{\partial u}{\partial y} & \frac{\partial u}{\partial z} \\
\frac{\partial v}{\partial x} & \frac{\partial v}{\partial y} & \frac{\partial v}{\partial z} \\
\frac{\partial w}{\partial x} & \frac{\partial w}{\partial y} & \frac{\partial w}{\partial z}
\end{array}\right]=\left[\begin{array}{ccc}
\lambda_{c r} & -\frac{1}{2} R & 0 \\
\frac{1}{2} R+\varepsilon & \lambda_{c r} & 0 \\
\xi & \eta & \lambda_{r}
\end{array}\right]
$$

where $u, v$ and $w$ are the three components of the velocity along the $x, y$, and $z$ directions in Cartesian Coordinate.

Since our purpose is to demonstrate the shear and stretching contaminations of different criteria numerically, we add shear and stretching components separately and calculate how different criteria respond. 


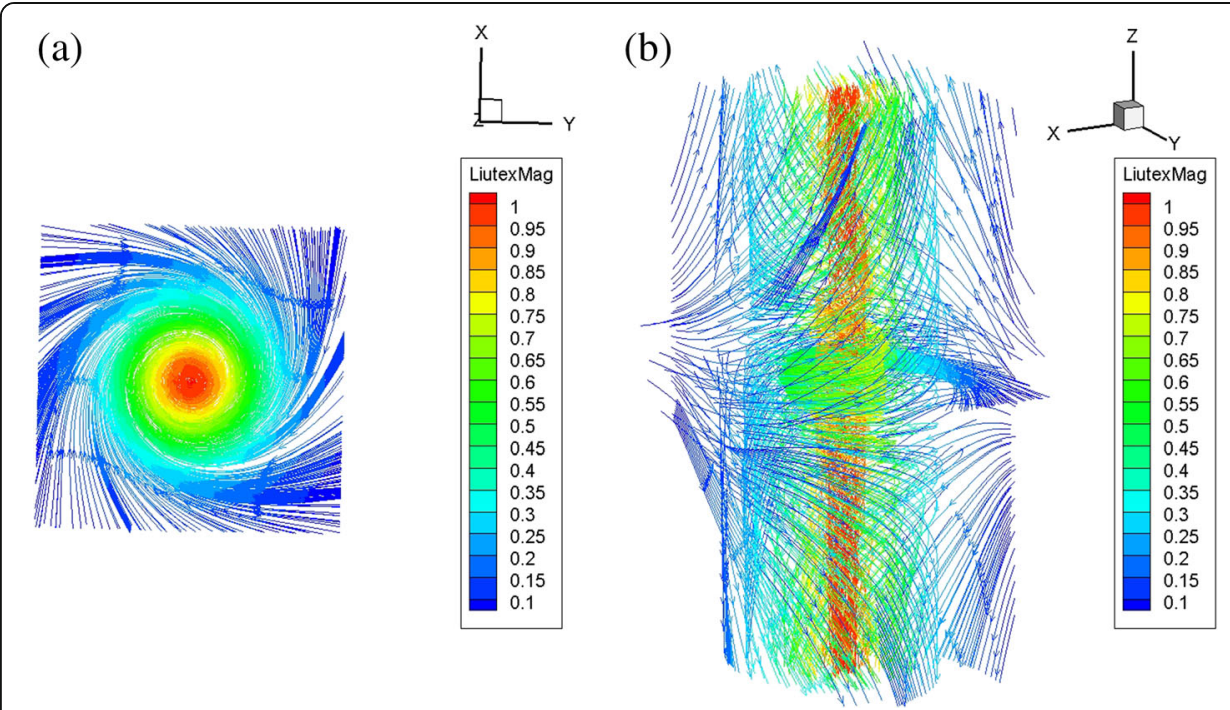

Fig. 1 (Color online) Streamlines of Burger vortex from top and side view with Liutex magnitude, which depicts the rotational strength of the fluid particles

\subsection{Adding shear components}

The matrix corresponding to shear is in the form of:

$$
\nabla \boldsymbol{V}_{\text {shear }}=\left[\begin{array}{ccc}
0 & 0 & 0 \\
\varepsilon_{a} & 0 & 0 \\
\xi_{a} & \eta_{a} & 0
\end{array}\right]
$$

The subscript " $a$ " refers to the "adding" of shearing components.

The new velocity gradient tensor in the Principal Coordinates after adding shear is

$$
\nabla V_{\mathbf{1}}=\left[\begin{array}{ccc}
\lambda_{c r} & -\frac{1}{2} R & 0 \\
\frac{1}{2} R+\varepsilon+\varepsilon_{a} & \lambda_{c r} & 0 \\
\xi+\xi_{a} & \eta+\eta_{a} & \lambda_{r}
\end{array}\right]
$$

Under the Principal Coordinates, the local rotation axis is the Z-axis, so, $\xi_{a}$ (value of $\frac{\partial w}{\partial x}$ component) and $\eta_{a}$ (value of $\frac{\partial w}{\partial y}$ component) are not in the rotation plane. Thus, these components will not influence the rotation strength. However, $\varepsilon_{a}$ is in the rotation plane, and thus affecting rotation strength. If $\varepsilon_{a}$ is in the direction of rotation, the Liutex magnitude remains unchanged, but if $\varepsilon_{a}$ is in the reverse direction of fluid rotation or negative and $\left|\varepsilon_{a}\right|>\varepsilon$, it may change the Liutex magnitude. This change occurred not because Liutex was contaminated by shear but because $\left|\frac{1}{2} R+\varepsilon+\varepsilon_{a}\right|<\frac{R}{2}$ which causes the strength of Liutex reduced according to the Liutex definition that the magnitude of rotation strength is $\min \left\{\left|\frac{\partial u}{\partial y}\right|_{\theta},\left|\frac{\partial v}{\partial x}\right|_{\theta}\right\}$ where $\left|\frac{\partial u}{\partial y}\right|_{\theta}$ and $\left|\frac{\partial v}{\partial x}\right|_{\theta}$ are the minimum absolute values of $\frac{\partial u}{\partial y}$ and $\frac{\partial v}{\partial x}$ respectively. Here we assume the rotation is anti-clockwise along the Z-axis. Therefore, when shear is in the opposite direction of rotation, $\min \{$ $\left.\left|\frac{\partial u}{\partial y}\right|_{\theta},\left|\frac{\partial v}{\partial x}\right|_{\theta}\right\}$ may change, affecting the Liutex magnitude. Following Proposition 1 gives the accurate measure about when shear may change the Liutex magnitude. 
Proposition 1: The rotation strength does not change if $\varepsilon_{a} \geq-\varepsilon$ where $\varepsilon>0$; the rotation strength changes if $\varepsilon_{a}<-\varepsilon$.

Proof: If $\varepsilon_{a} \geq-\varepsilon$, then $\left|\frac{1}{2} R+\varepsilon+\varepsilon_{a}\right| \geq\left|-\frac{1}{2} R\right|$, which shows that the Liutex magnitude is still $R$ which is defined as twice the angular speed of the rigid rotation.

- Remark: Physically, $\varepsilon_{a}<0$ means adding a shear against the rotation direction and $\varepsilon_{a}<-\varepsilon$ indicates that shear is strong to the extent that it can affect rotation strength. So, keeping the rotation strength the same, the shear matrix we add should satisfy the following requirement: $\varepsilon_{a} \geq-\varepsilon$. In other words, Proposition 1 just shows in some condition, external shear disturbance cannot change Liutex, but in other conditions $\left(\varepsilon_{a}<-\varepsilon\right)$, the external shear disturbance may. However, in any case, for a certain velocity gradient tensor, Liutex is always defined to represent the rigid rotation including the direction and strength. The definition has no shear and no stretching and cannot be considered as contaminated by shear or stretching.

\subsection{Adding stretching components}

The matrix corresponding to stretching is of the form

$$
\nabla V_{\text {stretching }}=\left[\begin{array}{ccc}
\alpha_{a} & 0 & 0 \\
0 & \beta_{a} & 0 \\
0 & 0 & \gamma_{a}
\end{array}\right]
$$

The subscript "a" means "adding" the stretching components.

The new velocity gradient tensor in the Principal Coordinate after adding stretching is

$$
\nabla \boldsymbol{V}_{\mathbf{2}}=\left[\begin{array}{ccc}
\lambda_{\mathrm{cr}}+\alpha_{a} & -\frac{1}{2} R & 0 \\
\frac{1}{2} R+\varepsilon & \lambda_{c r}+\beta_{a} & 0 \\
\xi & \eta & \lambda_{r}+\gamma_{a}
\end{array}\right]
$$

Proposition 2: The rotation strength does not change if $\alpha_{a}=\beta_{a}$.

Proof: Based on theorem 1, the rotation strength of $\nabla V_{2}$ is still $R$. Therefore, to keep the rotation strength unchanged, the added $\nabla V_{\text {stretching }}$ should satisfy $\alpha_{a}=\beta_{a}$.

- Remark: Proposition 1 and Proposition 2 just give some conditions under which Liutex may or may not change the magnitude and direction. They do not mean Liutex could be contaminated by shear or stretching for a certain velocity gradient tensor. Liutex is always a rigid rotation vector and never contaminated by any shear and stretching. On the other hand, look eqs. (43), (45), (48) and (54), all vorticity, $Q, \lambda_{c i}, \Delta$ are contaminated by shear or stretching or both where $R$ is defined as twice the angular speed of rigid rotation of fluids. The external shear may change Liutex under some conditions which means the external shear can change the rotation strength but does not mean Liutex is contaminated by shear. 


\subsection{Stretching contamination analysis}

The following procedure was implemented to depict the reaction of the vortex identification schemes over the change in stretching effects. Firstly, we select a point in Burgers vortex whose velocity gradient tensor is:

$$
\nabla \vec{V}_{B}=\left[\begin{array}{ccl}
-0.0419999994 & -0.0711665452 & 0.0000000000 \\
0.0711665452 & -0.0419999994 & 0.0000000000 \\
0.0000000000 & 0.0000000000 & 0.0839999988
\end{array}\right]
$$

Then for convenience, the stretching matrix $\nabla V_{\text {stretching }}$ is added and the values of $Q$, $\Delta, \lambda_{2}, \lambda_{c i}$, and Liutex are recorded where $\nabla V_{\text {stretching }}$ is given by (note that it satisfies the requirement of adding stretching):

$$
\nabla V_{\text {stretching }}=\left[\begin{array}{ccc}
0.02 & 0 & 0 \\
0 & 0.02 & 0 \\
0 & 0 & -0.04
\end{array}\right]
$$

The sum of diagonal elements of $\nabla V_{\text {stretching }}$ is equal to zero, so it satisfies the continuity equation of incompressible flow. The stretching effect given by $\nabla \boldsymbol{V}_{\text {stretch }}$ ing is increased repeatedly, step by step, and the values of every scheme are recorded. The results are presented in the following graph where the $\mathrm{x}$-axis represents the relative stretching rate (the ratio of stretching component and vorticity magnitude), and the $y$-axis gives the corresponding values of different vortex identification methods.

The change in stretching components results in a change in $Q, \Delta$, and $\lambda_{2}$. On the other hand, $\lambda_{c i}$ and $\mathrm{L}$ are not changed, indicating that $\lambda_{c i}$ and $\mathrm{L}$ are not contaminated by the stretching effect, as shown in Fig. 2. Further observation of Fig. 2 reveals that the increase in the stretching effect on $Q$ decreases its value after a certain point. The $\mathrm{Q}$ values ultimately become negative, indicating a non-vortical structure. However, other criteria indicate there is a vortex structure. So, it can be concluded that $Q$ may conflict with other criteria and violate the $\Delta$-rule. Theoretically, this is understandable as in Eq. (6), $\Delta$ could be positive even if $Q$ is negative provided that the square of the third variant is large enough. The computational results shown in Fig. 2 coincide with the theoretical analysis given by Eqs. (45), (48) and (54).

\subsection{Shear contamination analysis}

A similar procedure to the stretching effect is implemented for the graphic representation of the shearing effect on the vortex identification schemes. Again, for our convenience, the shearing matrix $\nabla V_{\text {shear }}$ is defined as:

$$
\nabla V_{\text {shear }}=\left[\begin{array}{ccc}
0 & 0 & 0 \\
0.02 & 0 & 0 \\
0.02 & 0.02 & 0
\end{array}\right]
$$

$\nabla V_{\text {shear }}$ is added to $\nabla \vec{V}_{B}$ a few times, and then the corresponding values of the different criteria are recorded. The results are presented in the following graph, where the $\mathrm{x}$-axis represents the relative shear rate, and the $\mathrm{y}$-axis gives the corresponding values of different vortex identification methods. 


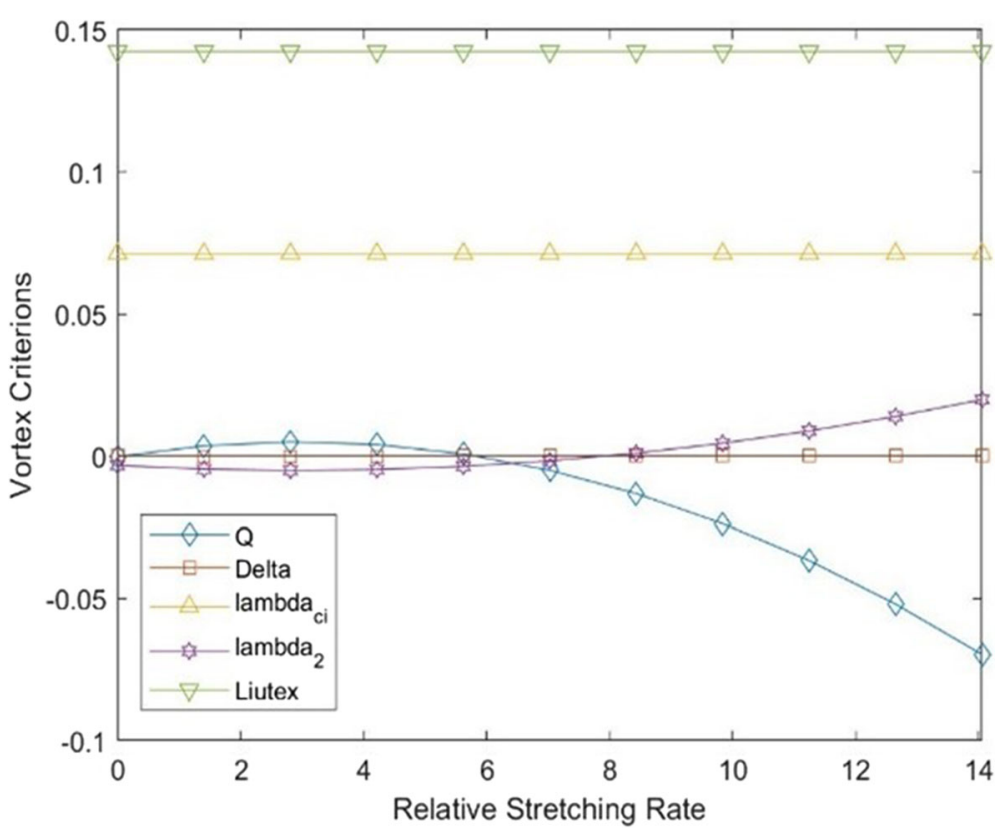

Fig. 2 Line graphs depicting the effect of stretching on different vortex identification methods

The relative shear rate is the ratio of the shearing component over vorticity magnitude. More precisely, it is the ratio of shear over vorticity at any point in our numerical domain. The reason we are dividing shear by vorticity is to refrain shear from getting too large. Fig. 3 indicates that $Q, \Delta, \lambda_{2}$, and $\lambda_{c i}$ are all affected by the change in the shearing component, while Liutex has remained unaffected by shear. It can be concluded from Fig. 2 and Fig. 3 that $Q, \Delta, \lambda_{2}$, and $\lambda_{c i}$ are affected by

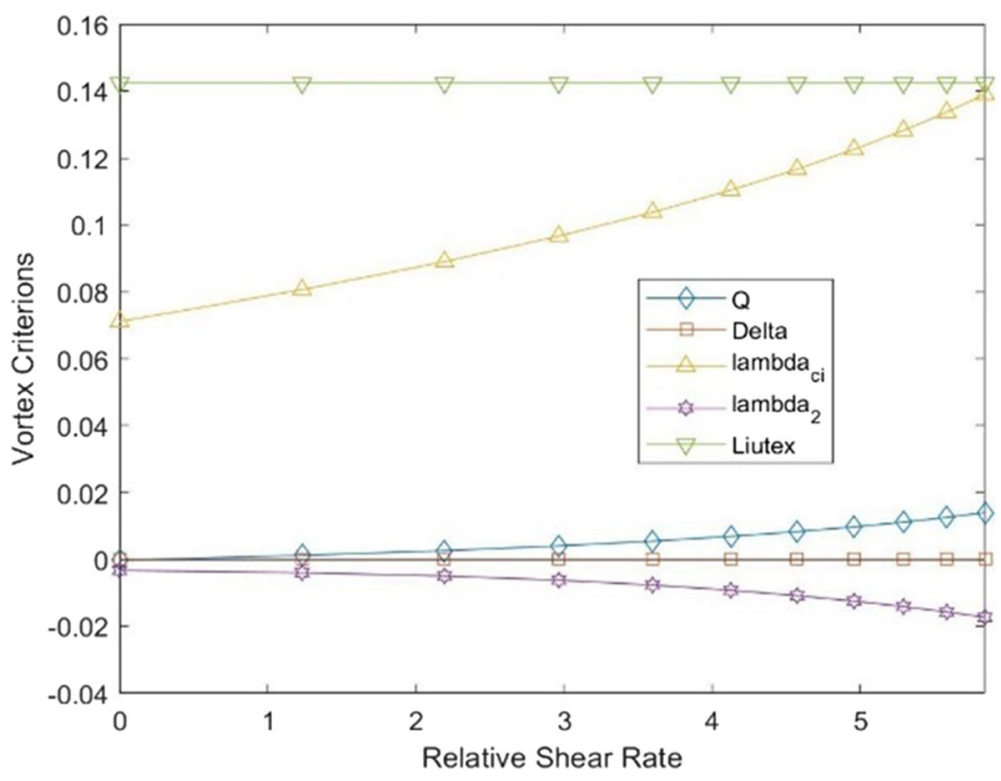

Fig. 3 Line graphs depicting the effect of shear on different vortex identification methods 

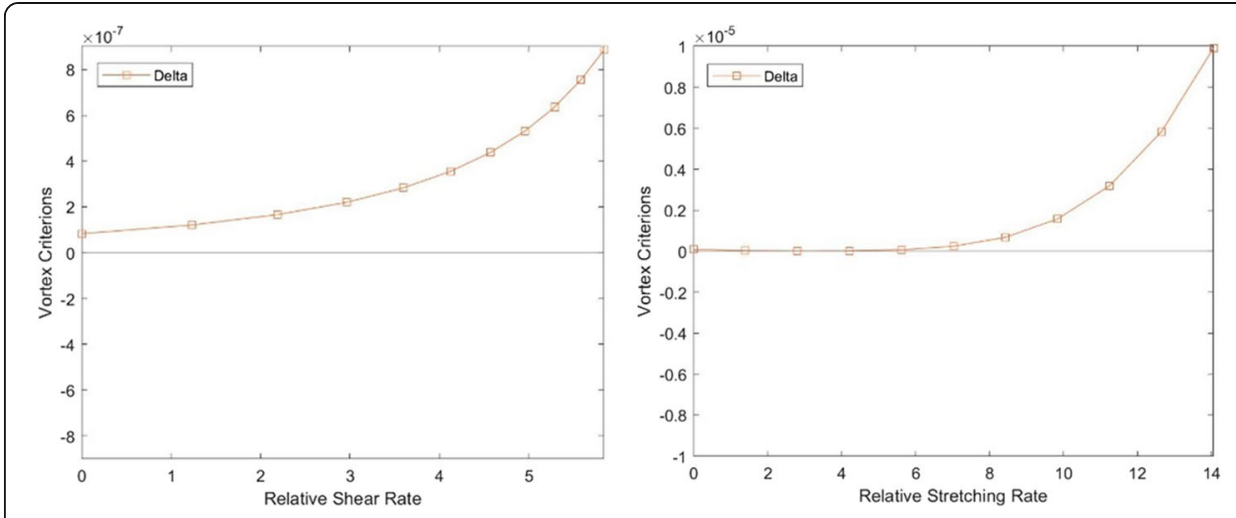

Fig. 4 Stretching and shearing effect on $\Delta$ method of vortex identification

either shear, stretching, or both at different levels, whereas the Liutex method is unaffected by both.

Since the values of $\Delta$ are very small, $\Delta$ looks to be consistently zero because $\Delta$ has sixth power of $R$ which is small; however, if we use zoom in, it can be observed that the $\Delta$ values are increasing. The $\Delta$ - plots are given in Fig. 4 below:

\section{Conclusion}

As the theoretical relation between Liutex and several 2G vortex identification methods are given, and a numerical example of Burger vortex is implemented, the following conclusions can be summarized.

1) Mathematical relations between Liutex and the $2 G$ vortex identification methods, such as $Q, \Delta, \lambda_{2}$ and $\lambda_{c i}$ methods are derived mathematically, which clearly show the second generation methods are severely contaminated by shearing, stretching, or by both. On the other hand, Liutex is the exact mathematical definition of fluid rotation or vortex.

2) The velocity gradient tensor in the Principal Coordinate can be uniquely decomposed into its rigid rotational part, stretching/compression part, and shearing part. This decomposition is known as the Liutex-based decomposition.

3) The $Q$ method conflicts with $\Delta, \lambda_{2}$, and $\lambda_{c i}$ methods, meaning that at some points, $\mathrm{Q}$ may mistreat the vortex point as a non-rotational point. Evidence of this can be seen in the graphical representations (see Fig. 2).

4) According to Eqs. (43), (45), (48), (54), we can have the following table (Table 1) as Liutex magnitude is defined as twice the angular speed of local rigid rotation.

5) The external shear may change Liutex under some conditions, especially when large shear force is applied in the opposite direction of rotation, which means the external shear can change the rotation strength but does not mean Liutex is

Table 1 Contamination by stretching and shearing on different criteria

\begin{tabular}{llllll}
\hline Methods & $\mathbf{Q}$ & $\boldsymbol{\Delta}$ & $\boldsymbol{\lambda}_{\mathbf{2}}$ & $\boldsymbol{\lambda}_{\boldsymbol{c i}}$ & $\mathbf{L}$ \\
\hline Contamination by stretching & Yes & Yes & Yes & No & No \\
Contamination by shearing & Yes & Yes & Yes & Yes & No \\
\hline
\end{tabular}


contaminated by shear, which can be seen clearly in the formula of the Liutex magnitude.

6) According to Table $1, Q, \Delta$, and $\lambda_{2}$ are stretch sensitive, while $Q, \Delta, \lambda_{2}$, and $\lambda_{c i}$ are affected by the shearing factor. The numerical results from the Burgers vortex coincide with the theoretical analysis. However, Liutex remained the same and is not affected. Therefore, we can conclude $Q, \lambda_{2}$, and $\lambda_{c i}$ methods may not appropriately represent vortex strength, while Liutex is the only one that correctly represents fluid rotation or vortex.

Acknowledgements

The authors are grateful to the Mathematical Department of the University of Texas at Arlington for housing the research team. The code "Liutex-UTA" is released by Prof. Chaoqun Liu in 2019. The authors thank TACC (Texas Advanced Computational Center) for providing the CPU hours.

\section{Authors' contributions}

PS performed the overall analysis of stretching and shearing effects of different vortex identification methods and was responsible for writing this manuscript. CN helped in sketching the graphs as well as proofreading and editing the manuscript. YY helped in proofreading and mathematical discussion about Principal Coordinates and Principal Tensor Decomposition. OA helped in coding to get the streamlines of Burger vortex. CL is the corresponding author of this paper who gave the original ideas and instructions in every step of the research and checked and modified the paper more than ten times. The authors read and approved the final manuscript.

\section{Author's information}

PS, CN, YY, and OA are all Ph.D. students of the Mathematics Department of UTA. CL is the Distinguished Professor and Director of the Center for Numerical Simulation and Modeling (CNSM) of UTA.

\section{Funding}

There is no funding to support this project.

\section{Availability of data and materials}

The data and materials that support the findings of this study are available from the corresponding author upon reasonable request.

\section{Competing interests}

The authors declare that they have no competing interests.

Received: 29 September 2020 Accepted: 28 December 2020

Published online: 12 March 2021

\section{References}

1. Liu C, Yan Y, Lu P (2014) Physics of turbulence generation and sustenance in a boundary layer. Comput Fluids 102:353-384

2. Helmholtz $\mathrm{H}$ (1858) On the integrals of the hydrodynamic equations corresponding to vortex motions. J für die reine und angewandte Mathematik 55:22-25 (in German)

3. Saffman P (1992) Vortices dynamics. Cambridge University Press, Cambridge

4. Zhou Y, Antonia RA (1993) A study of turbulent vortices in the near wake of a cylinder. J Fluid Mech 253:643-661

5. Epps B (2017) Review of vortex identification methods. AIAA 2017:0989

6. Robinson SK (1991) Coherent motion in the turbulent boundary layer. Annu Rev Fluid Mech 23:601-639

7. Liu C, Gao Y, Dong X, Wang Y, Liu J, Zhang Y, Cai X, Gui N (2019) Third generation of vortex identification methods: Omega and Liutex based systems. J Hydrodyn 31(2):205-223

8. Perry AM, Chong MS (1987) A description of eddying motions and flow patterns using critical-point concepts. Annu Rev Fluid Mech 19:125-155

9. Chong MS, Perry AM (1990) A general classification of 3-dimensional flow field. Phys Fluids A 2(5):765-777

10. Hunt JCR, Wray AA, Moin P (1988) Eddies, stream, and convergence zones in turbulent flows. Center for Turbulent Research Report CTR-S88, pp 193-208

11. Jeong J, Hussain F (1995) On the identification of a vortex. J Fluid Mech 285:69-94

12. Zhou J, Adrian R, Balachandar S, Kendall TM (1999) Mechanisms for generating coherent packets of hairpin vortices in channel flow. J Fluid Mech 387:353-396

13. Liu C, Wang Y, Yang Y, Duan Z (2016) New omega vortex identification method. Sci China Phys Mechan Astronomy 59(8):684711

14. Dong XR, Wang Y, Chen X, Dong Y, Zhang Y, Liu C (2018) Determination of epsilon for omega vortex identification method. J Hydrodyn 30(4):541-546

15. Zhang Y, Liu K, Li J, Xian H, Du X (2018) Analysis of the vortices in the inner flow of reversible pump-turbine with the new omega vortex identification method. J Hydrodyn 30(3):463469

16. Liu C, Gao Y, Tian S, Dong X (2018) Rortex- a new vortex vector definition and vorticity tensor and vector decompositions. Phys Fluids 30:035103

17. Gao Y, Liu C (2018) Rortex and comparison with eigenvalue-based identification criteria. Physics Fluid 30:085107 
18. Haller G (2005) An objective definition of the vortex. J Fluid Mech 525:1-26

19. Wang Y, Gao Y, Liu C (2018) Galilean invariance of Rortex. Phys Fluids 30:111701

20. Liu J, Wang Y, Gao Y, Liu C (2019) Galilean invariance of Omega vortex identification method. J Hydrodyn 31, 249-255. https://doi.org/10.1007/s42241-019-0024-2

21. Dong X, Gao Y, Liu C (2019) New normalized Rortex/vortex identification method. Phys Fluids 31(1):011701

22. Liu J, Liu C (2019) Modified normalized Rortex/vortex identification method. Phys Fluids 31(6):061704

23. Liu J, Gao Y, Liu C (2019) An objective version of the Rortex vector for vortex identification. Phys Fluids 31(6):065112

24. Gao Y, Liu C (2019) Rortex based velocity gradient tensor decomposition. Phys Fluids 31:011704

25. Gao Y, Yu Y, Liu J, Liu C (2019) Explicit expressions for Rortex tensor and velocity gradient tensor decomposition. Phys Fluids 31(8):081704

26. Yu Y, Shrestha P, Nottage C, Liu C (2020) Principal coordinates and principal velocity gradient tensor decomposition. J Hydrodyn 32:441-453. https://doi.org/10.1007/s42241-020-0035-z

27. Lamb H (1932) Hydrodynamics. Cambridge University Press, Cambridge

28. Chakraborty P, Balachandar S, Adrian R (2005) On the relationships between local vortex identification schemes. J Fluid Mech 535:189-214. https://doi.org/10.1017/S0022112005004726

29. Xu W, Gao Y, Deng Y, Liu J, Liu C (2019) An explicit expression for the calculation of the Rortex vector. Phys Fluids 31(9): 095102

30. Gao Y, Liu J, Yu Y, Liu C (2019) A Liutex based definition and identification of vortex core center lines. J Hydrodyn 31: 445-454. https://doi.org/10.1007/s42241-019-0048-7

31. Webster DR, Young DL (2015) A laboratory realization of the burgers' vortex cartoon of turbulence- plankton interactions. Assoc Sci Limnol Oceanogr Methods 13:92-102

32. Kolár V Sistek J (2019) Stretching response of Rortex and other vortex identification schemes. AIP Advance 9:105025

Publisher's Note

Springer Nature remains neutral with regard to jurisdictional claims in published maps and institutional affiliations.

Submit your manuscript to a SpringerOpen ${ }^{\circ}$ journal and benefit from:

- Convenient online submission

- Rigorous peer review

- Open access: articles freely available online

High visibility within the field

Retaining the copyright to your article

Submit your next manuscript at $>$ springeropen.com 